Revista Educación

ISSN: 0379-7082

ISSN: 2215-2644

revedu@gmail.com

Universidad de Costa Rica

Costa Rica

\title{
Inclusión laboral del profesorado adulto mayor con más años de estudio, en un panorama de envejecimiento poblacional hacia el 2050
}

Acero-Mondragón, Edward Javier

Inclusión laboral del profesorado adulto mayor con más años de estudio, en un panorama de envejecimiento poblacional hacia el 2050

Revista Educación, vol. 44, núm. 2, 2020

Universidad de Costa Rica, Costa Rica

Disponible en: http://www.redalyc.org/articulo.oa?id=44062184016

DOI: https://doi.org/10.15517/revedu.v44i2.39365

Esta obra está bajo una Licencia Creative Commons Atribución-NoComercial-SinDerivar 3.0 Internacional. 


\title{
Inclusión laboral del profesorado adulto mayor con más años de estudio, en un panorama de envejecimiento poblacional hacia el 2050
}

\author{
Inclusion of Senior Faculty with More Years of Academic Experience in the Workforce with an Outlook on the \\ Aging Population towards 2050
}

Edward Javier Acero-Mondragón

Universidad de La Sabana, Colombia

DOI: https://doi.org/10.15517/revedu.v44i2.39365

edward.acero@unisabana.edu.co

Redalyc: http://www.redalyc.org/articulo.oa?id=44062184016

iD http://orcid.org/0000-0002-1987-8716

Recepción: 23 Octubre 2019

Aprobación: 13 Mayo 2020

\section{Resumen:}

En el artículo se hace referencia a cómo en política económica mundial, las proyecciones del envejecimiento poblacional hacia el 2050, tienen como resultado aumentar la edad de jubilación, para disminuir la presión de las cargas gubernamentales fiscales y sanitarias. Sin embargo, en la persona adulta mayor con más años de estudios, se asocia con mayor estabilidad emocional y habilidad en la toma decisiones sobre todo si estas dependen de decisiones anteriores, aunado a que en el contexto educativo son valores deseables para formar nuevas generaciones. De esta manera, se señala que independientemente del deterioro sensitivo, sensorial y cognitivo que acompaña a algunas personas en el proceso de envejecimiento, adultos y adultas mayores con más años de estudios incluidos laboralmente como docentes, puede ser parte de una estrategia puntual para aumentar la proporción de ocupación de adultos mayores, aportando así a la sociedad y economía.

Palabras ClaVE: Docentes, Integración escolar laboral, Envejecimiento de la población.

\section{Abstract:}

The article references world economic policy projections regarding the aging population and the fact that by 2050 , the retirement age will increase in an effort to reduce government fiscal and healthcare burdens. However, older teaching faculty with more years of study are associated with greater emotional stability and better decision-making skills which are desirable traits for new generations of teachers in the educational context. Regardless of any sensory impairment which impact some aging individuals, many older adults who are more experienced and possess more years of study are still actively employed as educators. This may serve as a strategy to specifically increase the acceptance of employable older adults who can still provide valuable contributions to society and the economy.

KEYWORDS: Teachers, Integration of School Workforce, Aging Population.

\section{INTRODUCCIÓN}

El Informe sobre la Salud Global y Envejecimiento del Instituto Nacional del Envejecimiento de los Estados Unidos y la Organización Mundial de la Salud Suzman y Beard (2011) argumentan que

desde que se tienen datos demográficos los niños son más numerosos que las personas adultas mayores de 65 años, pero que impulsado por la caída de la tasa de fecundidad y aumento sustancial en la esperanza de vida, el envejecimiento de la población mundial va a acelerar previendo que puedan empezar a igualarse; y en proyección ya las personas mayores de 65 años se cree aumentarán pasando de un estimado de 524 millones en el 2010 a 1,5 mil millones en el 2050, con la mayor parte del aumento de esta población en los países en desarrollo. (p.2)

Respecto a países desarrollados, documentos en el ámbito público ya están haciendo análisis sobre el envejecimiento demográfico y sus derivaciones sociales, centrándose principalmente sobre su impacto fiscal en las estructuras económicas y sanitarias; por ejemplo sobresalen los del Consejo Nacional de Investigación de Estados Unidos, plasmados en el libro llamado Preparando un mundo envejecido, en el cual "se advierte 
lo que implica sobre los reajustes fiscales en la carga pensional y el sistemas de salud" (Moore, 2001, p. 200); por su parte la Unión Europea, con la comisión Salud y Protección al Consumidor en el documento para un Envejecimiento Saludable: Claves para una Europa Sustentable, advierten también sobre la disminución de la fuerza laboral analizando entre otros la carga prestacional de tipo pensional que esto conlleva (Siddall, Kjaeserud, Dziworski, Przywara y Xavier, 2007) En el ámbito privado, organizaciones no gubernamentales también hacen el mismo enfoque; por ejemplo el Centro Europeo para Políticas de Cuidado Social e Investigación de Viena, Austria (Zaidi 2008) y el Programa Global de Envejecimiento Demográfico de la Universidad de Harvard en Estados Unidos (Beard, Biggs, Bloom, Fried y Hogan, 2012) cuentan cómo el envejecimiento de la población genera muchos desafíos y preocupaciones sobre el ritmo del crecimiento económico, los sistemas de pensiones y el funcionamiento e integridad financiera de la asistencia sanitaria; $y$ publicaciones independientes sobre envejecimiento demográfico también en estos países, sistemáticamente hacen la misma aproximación política en lo económico (De la Croix, Pierrard y Sneessens, 2010; Bloom et al., 2015; Epure, 2012), y en política sanitaria (Wiener y Tilly, 2002; Gray, 2005) (Ansah et al., 2014).

En Latinoamérica, un primer estudio referente sobre envejecimiento poblacional como el de Brea (2003), plantea que entre los años 2000 a 2050, la población de 65 años incrementará entre 10 a $27 \%$ en Cuba y del 5 al 18\% en Brasil; de la misma forma un estudio referente en envejecimiento poblacional Latinoamericano proyectado como el de Leeson (2011) muestra entre otros que para Guatemala aumentará en un 10\% su población por encima de 65 años al año 2050, relatando además que en el año 1980 en Latinoamérica alrededor del 40\% de la población era menor de 15 años y en el años 2010 paso a ser menos del 30\%, y que mientras los mayores de 65 años hoy están alrededor del 7\%, eran menos del 5\% en el siglo veinte. En esta parte del mundo documentos públicos como los de la División de Población de la Comisión Económica para América Latina y el Caribe (CEPAL) editado por Huenchuan (2010), documentan estrategias para asumir los cambios involucrados por el envejecimiento poblacional latinoamericano, no haciendo diferentes análisis de aquellos estudios globales al respecto que se centran también en las dimensiones de política económica y sanitaria. Con este contexto y como dice Christensen, Doblhammer, Rau, y Vaupel (2009) se entiende que

la mayoría de los gobiernos se mueva en la dirección económica aumentando la edad de jubilación y sanitaria mejorando el sistema de salud, pues son estrategias razonables para hacer frente a las implicaciones del envejecimiento de la población, pero estas estrategias deben ir junto con cambios en políticas de empleo, que involucren ocupación de puestos de trabajo que requieran conocimiento, lo que implica aumentar la proporción de personas que en sus 60 y 70 años son capaces de contribuir a la economía. (p.10)

El propósito del texto que se presenta es hacer ver que en un panorama de envejecimiento de la población hacia 2050, independientemente del deterioro sensitivo, sensorial y cognitivo asociado, en personas adultas mayores con más años de estudio hay valores que en un marco de inclusión laboral como profesores, puede ser parte de una estrategia para aumentar la proporción de ocupación de adultos y adultas mayores que contribuyen a la sociedad y economía.

\section{EL ENVEJECIMIENTO DE LA POBLACIÓN: DÉFICITS FÍSICOS Y COGNITIVOS ASOCIADOS}

Revisiones sistemáticas sobre el envejecimiento, plantean que es un proceso biológico normal (Kunlin, 2010; Teixeira y Guariento, 2010; Trindade et al., 2013), que lleva consigo reconocidos cambios como déficits del control motor, sensitivo, sensoriales y cognitivos; en ejemplo de déficits de control motor Levin, Fujiyama, Boisgontier, Swinnen, y Summers (2014) explica ampliamente cómo en hombres adultos saludables de alrededor los 70 años con estimulación motora transcraneal declina el desempeño del control de los movimientos voluntarios; y en ejemplo de déficits de control sensitivo, Wickremaratchi y Llewelyn (2006) revisan como al tacto por estimulación en rangos de vibración entre 40 y $250 \mathrm{~Hz}$ están disminuidos en personas adultas entre 55 a 84 años respecto aquellos entre 19 y 31 años. En ejemplo de déficits de control 
sensorial Boyce y Shone (2006) cuentan cómo el olfato declina especialmente a partir de la séptima década de vida, más rápido que el gusto; y acerca del sentido del oído Cruickshanks, Margarete y Wichmann (2012) relatan cómo en un estudio sobre 3285 personas entre 21 a 84 años se halló que el $42.7 \%$ de aquellos que se encontraban en los rangos de edad entre 65 a 84 años tuvieron discapacidad auditiva, con la particularidad de que por cada cinco años de edad, la razón de relación o -OR- de la discapacidad auditiva aumentaba a un OR de 1.80; en cuanto a la visión Chader y Taylor (2013) exponen que las personas a partir de los 65 años incrementan la degeneración macular, lo que disminuye la agudeza visual; y respecto al déficit cognitivo un estudio focal prospectivo de Boyle et al. (2012) hace notar con métodos de encuesta prediseñada con el objetivo de encontrar susceptibilidad a la estafa económica, que cuando se aplicó a 420 personas saludables con promedio de edad de 83.5 años -desviación estándar 7,4 años sin demencia y sin enfermedad de Alzheimer, se dificulta la toma de decisiones incluso sin disfunción cognitiva manifiesta, lo que los hace susceptibles al engaño; quizá porque como deja ver un estudio experimental focal prospectivo hecho por Tymula, Belmaker, Ruderman, Glimcher y Levy (2013) con un juego económico aplicado a una población urbana de 135 personas saludables agrupadas por edades entre 12 y 17 años, 21 a 25 años, 30 a 50 años y 65 a 90 años, los adultos mayores al envejecimiento normal respecto a los más jóvenes, tienen comprometida la habilidad para decidir de forma individual de manera ventajosa, por tener menores expectativas económicas (Denburg, Tranel y Bechara, 2005; Fein, McGillivray y Finn, 2007; Rogalsky, Vidal, Li y Damasio (2012).

\section{ENVEJECIMIENTO Y LA FUNCIÓN EJECUTIVA SUPERIOR CON MÁS AÑOS DE EDUCACIÓN}

Un estudio experimental clásico hecho por Leibovici, Ritchie, Ledesert y Touchon (1996) con 283 personas de las cuales 87 fueron hombres y 196 mujeres con edad promedio de 74.7 y desviación estándar de siete años, comparó el desempeño en varios dominios cognitivos con respecto a los años de estudio de cada participante, demostrando que para las dimensiones cognitivas de habla, lenguaje, y atención, así como para la memoria secundaria, quienes tenían hasta educación universitaria o altos niveles de estudio o más años de educación, tuvieron mejores desempeños comparados con quienes tenían hasta educación primaria o bajos niveles de estudio o menos años de educación; evidencia que demostró cómo la formación educativa o años de educación previa contribuyen a atenuar el declive cognitivo dentro del curso progresivo del envejecimiento normal, lo cual fue notado por Andreoletti y Lachmanen (2004) en un pequeño estudio con 149 personas adultas entre 21 y 80 años y también en un amplio estudio norteamericano elaborado por Reuser, Willekens y Bonneux (2011) con 22388 participantes entre 55 y 95 años. Ahora en cuanto a la cognición en relación con los años de educación en adultos mayores los estudios han ido más allá, demostrando como el control ejecutivo que involucra un amplio rango de actividades que se envuelven en la planeación, organización, coordinación, implementación, y evaluación de muchas de las actividades rutinarias tienen menor deterioro al envejecimiento en personas con más años de educación, respecto a aquellos con menos años de educación (Hamdan, Mara y Hamdan, 2009; Voos, Custódio y Malaquias, 2011; Agrigoroaei, y Lachman, 2011);. En escenarios laborales no hay investigaciones sobre desempeño de los dominios cognitivos en relación con los años de educación o nivel de estudios en personas adultas mayores, quizá porque como revisa Sauré y Zoabi (2011) el retiro laboral al menos en países de Occidente, para los hombres trabajadores varía ampliamente entre México con 75 años a Bulgaria con 58,2 años, con promedio de 66,6 años y amplia desviación estándar; sin embargo, ejemplos en marcos laborales con gente adulta no mayor que cruza entre otras las variables demográficas de edad y nivel de estudios, encuentran que quienes tienen más edad y más años de educación se relacionan fuertemente con su posición en la empresa, y en esa posición, además influenciando un estilo de liderazgo (Craven y Kao, 2006; Ryan, Tavitiyaman y Weerakit, 2009; Ekaterini, 2010) 


\section{LA INCLUSIÓN}

En el informe de investigación para el organismo del Gobierno de España Instituto de Mayores y Servicios Sociales (IMSERSO) sobre envejecimiento productivo elaborado por Triadó, Celdrán, Conde, Montoro y Villar (2008) se explica la posibilidad de seguir trabajando más allá de la edad estimada para la jubilación mientras la esperanza de vida crezca, e invita a que conceptos tradicionales

como el de la 'necesidad de jubilarse' a una edad determinada deberán ser reconsiderados en diferentes niveles, uno de ellos desde las políticas públicas y otros hasta las prácticas de las empresas, y hace ver que en el entorno europeo a nivel laboral se está flexibilizando el concepto de jubilación de modo que no se prescinda de manera radical de las aportaciones de los trabajadores de más edad. (p.15)

Existe un cierto consenso en la sociedad de que la sabiduría de la persona adulta mayor implica el uso de ciertos tipos de razonamiento pragmático que permiten navegar en los importantes desafíos de la vida social (Grossmann et al., 2010); una sólida revisión de Mather y Cartensen, (2005) muestra evidencia asociada de que al envejecimiento, la gente adulta mayores hace mejor regulación de sus emociones. Si a estos aspectos cognitivos asociados al envejecimiento se suman resultados como los de Worthy, Gorlick, Pacheco, Schnyer y Maddox, (2011), que en un estudio comparó 28 adultos y adultas mayores de 60 a 84 años con 17.28 años promedio de educación y 28 personas adultas jóvenes de 18 a 23 años con 15.34 promedio de educación, encontrando que en toma decisiones cuando hay varias opciones posibles la gente adulta es mejor que la gente joven y sobre todo cuando dependen de decisiones anteriores; resultados corroborados con un estudio posterior con 58 adultos/as mayores entre 60 a 82 años y 56 jóvenes entre 18 a 23 años (Worthy y Maddox, 2012); entonces puede explorarse la inclusión del profesorado adulto mayor con más años de estudio en escenarios educativos, porque allí un inherente rol del conocimiento, sabiduría y emociones controladas encuentra el espacio para ejemplificar cómo las funciones ejecutivas superiores se involucran en la toma de decisiones; paralelamente como propone Christensen et al. (2009) respecto a involucrar a la persona adulta mayor con conocimiento al trabajo, un aporte a la economía y a la sociedad.

Moller (2005) como Veugelers (2011) exponen que la educación es una empresa moral, basada en principios y valores democráticos; entonces y en esa dirección el profesorado adulto mayor hacia los 60 a 70 años los propende, debido a su naturaleza cognitiva, deseable y esperada por la sociedad, más cuando se busca formar nuevas generaciones

En la educación latinoamericana desde las políticas públicas hasta las prácticas de las empresas educativas carece de investigaciones con personas adultas mayores que crucen variables demográficas como su edad y nivel de estudios logrados con su liderazgo y el impacto de sus decisiones en el desempeño académico y/o logros de sus estudiantes, entonces con este enfoque en políticas de envejecimiento poblacional mirando al año 2050, tienen en la posición del profesorado adulto mayor con más años de educación, estrategias públicas o privadas concretas para aumentar la proporción de ocupación de personas con conocimiento entre 60 y 70 años de edad, y de esta manera aportar en doble vía a la economía y sociedad.

\section{Conclusión}

Con el envejecimiento se asocia y reconoce el surgimiento de déficits físico-sensoriales, sensitivos y motores; sin embargo en los ámbitos cognitivos si bien se describe también un declive, este es atenuado acorde a los años o nivel de estudios en los dominios del habla, lenguaje, atención, memoria secundaria, así como en el control ejecutivo rutinario de planeación, organización, coordinación, implementación, y evaluación. Además, con el proceso de envejecimiento también es reconocido que se incorporan cognitivamente la sabiduría, especialmente para los desafíos de la vida social, control de emociones y mejor toma de decisiones cuando dependen de otras tomadas anteriormente. Por ello, políticas de empleo en educación con inclusión 
de fuerza laboral de docentes adultos/as mayores sobre los 60 a 70 años mirando hacia el 2050, encuentran en los espacios académicos estrategias públicas o privadas para hacerlo, porque siendo la educación una empresa moral, basada en principios y valores democráticos, es desde esta posición que se puede propender por esos valores, más cuando se forman nuevas generaciones.

\section{ReFERENCIAS}

Agrigoroaei, S., y Lachman, M. (2011). Cognitive functioning in midlife and old age: combined effects of psychosocial and behavioral factors. The Journals of Gerontology, 66(Suppl 1), i130-i140. doi: https://doi.org/10.1093/ger onb/gbr017

Andreoletti, C. y Lachman, M. (2004). Susceptibility and resilience to memory aging stereotypes: Education matters more than age. Experimental Aging Research, 30, 129-148.doi: https://doi.org/10.1080/03610730490274167

Ansah, J., Eberlein, R., Love, S., Bautista, M., Thompson, J. Malhotra, R. y Matchar, D. (2014). Implications of longterm care capacity response policies for an aging population: A simulation analysis. Health Policy, 116(1), 105-13 doi: https://doi.org/10.1016/j.healthpol.2014.01.006

Beard, J., Biggs, S., Bloom, D., Fried, L. y Hogan, P. (2012). Population aging: Facts, hallenges, and Responses. Suiza: World Economic Forum. Recuperado de https://cdn1.sph.harvard.edu/wp-content/uploads/sites/1288/2013 /10/PGDA_WP_89.pdf

Bloom, D., Chatterji, S., Kowal, P., Lloyd-Sherlock, P., McKee, M., Rechel, B., Rosenberg, L. y Smith. J. (2015). Macroeconomic implications of population ageing and selected policy responses. Lancet 385(9968), 649-657. doi: https://doi.org/10.1016/S0140-6736(14)61464-1

Boyce, J. y Shone, G. (2006). Effects of ageing on smell and taste. Postgraduate Medical Journal, 82(996), 239-241. doi: https://doi.org/10.1136/pgmj.2005.039453

Boyle, P., Yu, L., Wilson, R., Gamble, K., Buchman, A. y Bennett, D. (2012). Poor Decision Making Is a Consequence of Cognitive Decline among Older Persons without Alzheimer's Disease or Mild Cognitive Impairment. PLOS One, 7(8), 1-5. doi: https://doi.org/10.1371/journal.pone.0043647

Brea, J. (2003). Population Dynamics in Latin America. Population Bulletin 58(1), 1-38. Recuperado de https://bit .ly/2KOrIOy

Chader, G. y Taylor, A. (2013). Preface: The aging eye: normal changes, age-related diseases, and sight-saving approaches. Invest Ophthalmol Vis Sci., 54(14),1-4. doi: https://doi.org/10.1167/iovs.13-12993

Christensen, K., Doblhammer, G., Rau, R. y Vaupel, J. (2009). Ageing populations: the challenges ahead. Lancet. 374(9696), 1196-1208. doi: https://doi.org/10.1016/S0140-6736(09)61460-4

Craven, A. y Kao, J. (2006). The Relationship Between Leadership Style y Demographic characteristics Of Taiwanese Executives. International Business y Economics Research Journal. 5(2), 35-48. doi: https://doi.org/10.19030/ibe r.v5i2.3456

Cruickshanks, K., Margarete, A. y Wichmann, B. (2012). Hearing impairment and other health conditions in older adults: Chance associations or opportunities for prevention?. Semin Hear, 33(03), 217-224. doi: https://doi. org/10.1055/s-0032-1315720

De la Croix, D., Pierrard, O. y Sneessens, H. (2010). Aging and pensions in general equilibrium: Labor market imperfections matter. Institute for the Study of Labor (IZA) Discussion Papers, 5276., 1-35. Recuperado de htt p://hdl.handle.net/10419/4599

Denburg, N., Tranel, D. y Bechara, A. (2005). The ability to decide advantageously declines prematurely in some normal older persons. Neuropsychologia, 43(7), 1099-1106. doi: https://doi.org/10.1016/j.neuropsychologia.2 004.09 .012

Ekaterini, G. (2010). The Impact of Leadership Styles on Four Variables of Executives Workforce. International Journal of Business and Management 5(6), 3-16. doi: https://doi.org/10.5539/ijbm.v5n6p3 
Epure, M. (2012). Population ageing - a demographic trend with various consequences. Review of Applied SocioEconomic Research, 4(2), 97-107. Recuperado de https://bit.ly/3d6SFsC

Fein,G., McGillivray, S. y Finn,P. (2007). Older Adults Make Less Advantageous Decisions than Younger Adults: Cognitive and Psychological Correlates J. Int. Neuropsychol Soc., 13(3), 480-489. doi:https://doi.org/10.1017 /S135561770707052X

Gray, A. 2005. Population Ageing and Health Care Expenditure. Ageing Horizons, 2, 15-20. Recuperado de https:/ /www.ageing.ox.ac.uk/download/15

Grossmann, I., Naa, J., Varnuma, M., Parkb, D., Kitayamaa, S. y Nisbetta, R. (2010). Reasoning about social conflicts improves into old age. Proc Natl Acad Sci, 107(16), 7246-7250. doi: https://doi.org/10.1073/pnas.1001715107

Hamdan, A., Mara, E. y Hamdan, R. (2009). Effects of age and education level on the Trail Making Test in a healthy Brazilian sample. Psychology y Neuroscience. 2(2),199-203. doi: https://doi.org/10.3922/j.psns.2009.2.012

Huenchuan, S. (2010). Ageing, Human Rights and Public Policies. Chile: CEPAL. Recuperado de https://bit.ly/2K Q23Vm

Kunlin, J. (2010). Modern Biological Theories of Aging. Aging Dis. 1(2), 72-74. Recuperado de https://www.ncbi.n lm.nih.gov/pmc/articles/PMC2995895/

Leeson, G. (2011). The Demographics of Population Ageing in Latin America, the Caribbean and the Iberian Peninsula, 1950-2050. Oxford: University of Oxford. Recuperado de https://www.ageing.ox.ac.uk/files/larna_demograp hics.pdf

Leibovici, D., Ritchie, K., Ledesert, B. y Touchon, J. (1996). Does Education Level Determine the Course of Cognitive Decline?. Age and Ageing 25(5), 392-397. doi: https://doi.org/10.1093/ageing/25.5.392

Levin, O., Fujiyama, H., Boisgontier, M., Swinnen, S. y Summers, J. (2014) Aging and motor inhibition: A converging perspective provided by brain stimulation and imaging approaches. Neurosci Biobehav Rev, 43(12), 100-117. doi:https://doi.org/10.1016/j.neubiorev.2014.04.001

Mather, M. y Cartensen, L. (2005). Aging and motivated cognition: the positivity effect in attention and memory. Trends Cogn Sci, 9(10), 496-502. doi: https://doi.org/10.1016/j.tics.2005.08.005

Moller, J. (2005). Framing Successful School Leadership as a Moral and Democratic Enterprise. Education Research and Perspectives. 32(2), 43-56. Recuperado de https://bit.ly/3aQSos3

Moore, E. (2001). The Health of Aging Populations. Washington, D.C.: National Academies Press. doi: https://doi. org/10.17226/10120

Reuser, M., Willekens, F. y Bonneux, L. (2011). Higher education delays and shortens cognitive impairment. A multistate life table analysis of the US Health and Retirement Study. Eur J Epidemiol. 26(5), 395-403. doi: ht tps://doi.org/10.1007/s10654-011-9553-x

Rogalsky, C., Vidal, C., Li., X. y Damasio, H. (2012). Risky Decision-making in Older Adults without Cognitive Deficits: an fMRI study of VMPFC using the Iowa Gambling Task. Soc Neurosci, 7(2),178-190. doi: https:// doi.org/10.1080/17470919.2011.588340

Ryan, B. Tavitiyaman, P., Weerakit, N. (2009).The impact of gender, age and education related to leadership competencies needed for success as a hotel general manager. International CHRIE Conference-Refereed Track, 6, 1-8. Recuperado de https://scholarworks.umass.edu/refereed/Sessions/Saturday/6

Sauré, P. y Zoabi, H. (2011). Retirement Age Across Countries: The Role of Occupations. New York. USA: SSRN. Recuperado de https://papers.ssrn.com/sol3/papers.cfm?abstract_id=1940452

Siddall C, Kjaeserud, G., Dziworski, Przywara, B. y Xavier, A. (2007). Healthy ageing, a keystone for a sustainable Europe. Brussels: HSP. Recuperado de https://www.uv.es/ atortosa/healthyageingEU

Suzman, R. y Beard, J. (2011). Global Health and Aging. New York, USA: ONU -WHO. Recuperado de https://w ww.who.int/ageing/publications/global_health.pdf

Triadó, C., Celdrán, M., Conde, L.V., Montoro, J. y Villar, V. (2008). Envejecimiento productivo\#: productivo\#: la provisión de cuidados de los abuelos a implicaciones para su salud y bienestar. Barcelona: Instituto De Mayores Y Servicios Sociales. Recuperado de https://bit.ly/3faq $47 \mathrm{~h}$ 
Trindade, L., Aigaki, T., Peixoto, A., Balduino,A. DaCruz, I. y Heddle, J. (2013). A novel classification system for evolutionary aging theories. Front Genet, 25(4), 1-8. doi: https://doi.org/10.3389/fgene.2013.00025

Teixeira, I. y Guariento,M. (2010). Biology of aging: theories, mechanisms, and perspectives, 15(6), 2845-57. doi: https ://doi.org/10.1590/s1413-81232010000600022

Tymula, A., Belmaker, R., Ruderman. L., Glimcher, P. y Levy I. (2013) Like cognitive function, decision making across the life span shows profound age-related changes. Proc Natl Acad Sci, 110(42), 17143-17148. doi: https://doi .org/10.1073/pnas.1309909110

Veugelers W.(Eds.) (2011) Education and Humanism. Moral Development and Citizenship Education. Springer: Sense Publishers. Recuperado de https://doi.org/10.1007/978-94-6091-577-2_1

Voos, M., Custódio, E. y Malaquias, J. (2011). Relationship of Executive Function and Educational Status With Functional Balance in Older Adults. Journal of Geriatric Physical Therapy, 1(34),11-18. doi: https://doi.org/1 $0.1097 / J P T .0 b 013 \mathrm{e} 3181 \mathrm{ff} 2452$

Wickremaratchi, M., y Llewelyn, J. (2006). Effects of ageing on Touch. Postgrad Med J, 967(82),301-304. doi: http s://doi.org/10.1136/pgmj.2005.039651

Wiener, J. y Tilly, J. (2002) Population ageing in the United States of America: implications for public programmes, International Journal of Epidemiology, 31(4),776-781.doi: https://doi.org/10.1093/ije/31.4.776

Worthy, D., Gorlick,M., Pacheco, J., Schnyer, D. y Maddox, W. (2011). With Age Comes Wisdom: Decision-Making in Younger and Older Adults. Psychol Sci. 22(11),1375-1380. doi: https://doi.org/10.1177/0956797611420 301

Worthy, D. y Maddox,T. (2012). Age-based differences in strategy use in choice tasks. Frontiers in Neuroscience, 145(5),1-10. doi: https://doi.org/10.3389/fnins.2011.00145

Zaidi, A. (2008). Features and Challenges of Population Ageing: The European Perspective. Policy Brief 1, 1-16. Recuperado de https://core.ac.uk/download/pdf/11872350.pdf

\section{BY-NC-ND}

